\title{
Fast and Robust Disparity Estimation from Noisy Light Fields Using 1-D Slanted Filters
}

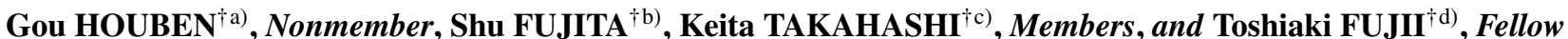

SUMMARY Depth (disparity) estimation from a light field (a set of dense multi-view images) is currently attracting much research interest. This paper focuses on how to handle a noisy light field for disparity estimation, because if left as it is, the noise deteriorates the accuracy of estimated disparity maps. Several researchers have worked on this problem, e.g., by introducing disparity cues that are robust to noise. However, it is not easy to break the trade-off between the accuracy and computational speed. To tackle this trade-off, we have integrated a fast denoising scheme in a fast disparity estimation framework that works in the epipolar plane image (EPI) domain. Specifically, we found that a simple 1-D slanted filter is very effective for reducing noise while preserving the underlying structure in an EPI. Moreover, this simple filtering does not require elaborate parameter configurations in accordance with the target noise level. Experimental results including real-world inputs show that our method can achieve good accuracy with much less computational time compared to some state-ofthe-art methods.

key words: light field, epipolar plane image, denoising, disparity estimation

\section{Introduction}

Due to recent advances in image acquisition techniques [1][5], depth (disparity) estimation from a set of multi-view images or a light field has attracted much research interest. The most straightforward approach is called multi-view stereo [6]-[12], where the classical stereo matching methods for two images are extended directly to a set of multi-view images. The basic idea is to find corresponding points across the images. Defocus and correspondence cues [13], [14], which were recently developed for light fields, also fall into this category. Another approach [15]-[20] is to analyze the structure of an epipolar plane image (EPI) [21]-[23] that is obtained from the light field. This approach is based on the fact that an EPI consists of many line patterns, and the slopes of those lines are directly related to the depth information. Wanner and Goldluecke [15], [19] have applied structure tensor analysis to EPIs to estimate the slopes. Their method has been proven to be fast and accurate when the light field is sufficiently dense, i.e., the disparity range between the neighboring viewpoints is sufficiently small, as is often the case with those taken by light field cameras [24].

Manuscript received February 6, 2019.

Manuscript revised May 20, 2019.

Manuscript publicized July 3, 2019.

${ }^{\dagger}$ The authors are with the Graduate School of Engineering, Nagoya University, Nagoya-shi, 464-8603 Japan.

a) E-mail: houben@fujii.nuee.nagoya-u.ac.jp

b) E-mail: s.fujita@fujii.nuee.nagoya-u.ac.jp

c) E-mail: keita.takahashi@nagoya-u.jp

d) E-mail: fujii@nuee.nagoya-u.ac.jp

DOI: $10.1587 /$ transinf.2019PCP0003
In this paper, we focus on how to handle a dense but noisy light field for disparity estimation, because if left as it is, the noise degrades the accuracy of estimated disparity maps. Several researchers have worked on this problem, e.g., by introducing disparity cues that are robust to noise [25], [26]. However, it is not easy to break the tradeoff between the accuracy and computational speed. To tackle this trade-off, we proposed a method in [27]*, which combines a fast disparity estimation framework developed in [19] with a fast denoising scheme. Specifically, we found that a simple 1-D slanted filter is very effective for reducing noise while preserving the underlying structure in an EPI. Moreover, this simple filtering does not require elaborate parameter configurations in accordance with the target noise level. Experimental results including real-world inputs show that our method can achieve good accuracy with much less computational time compared to some state-ofthe-art methods.

In the remainder of this paper, we first mention disparity estimation through the EPI analysis in Sect. 2. We will then introduce our method that incorporates denoising with 1-D slanted filters into the framework of the EPI analysis in Sect. 3. Experimental validations are presented in Sect. 4, followed by conclusion in Sect. 5 .

\section{Disparity Estimation through EPI Analysis}

We assume that a set of multi-view images, such as that shown in Fig. 1 (left), is given. These images constitute a 4-D light field $l(s, t, x, y)$, where $(s, t)$ denotes a viewpoint and $(x, y)$ denotes a pixel position. A 2-D subspace of the 4$\mathrm{D}$ light field with a fixed $(s, x)$ or $(t, y)$ is called an epipolar

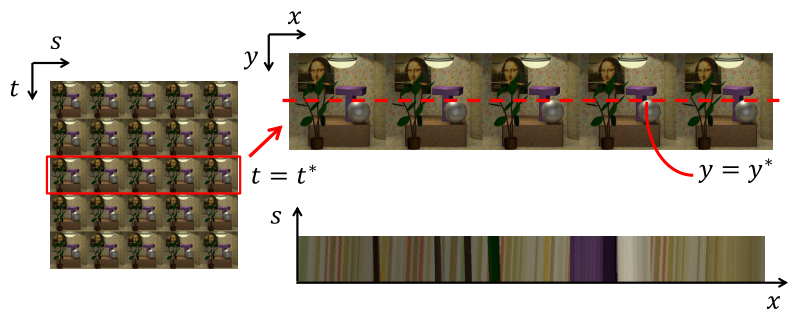

Fig. 1 Multi-view images (left) and EPI (right)

${ }^{*}$ This paper is an extension of our conference paper [27], with more thorough experimental evaluations. Several experimental results were revised, because we noticed that some conditions were not consistent among different methods in [27]. 


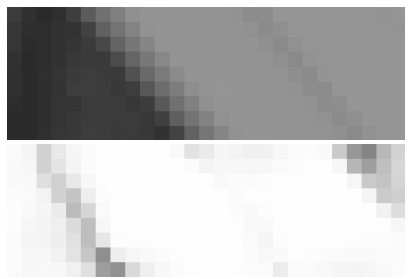

(a) Noiseless EPI

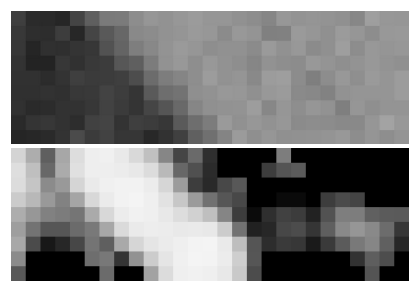

(b) Noisy EPI

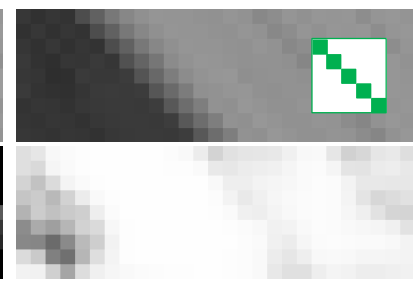

(c) Denoising along a slope

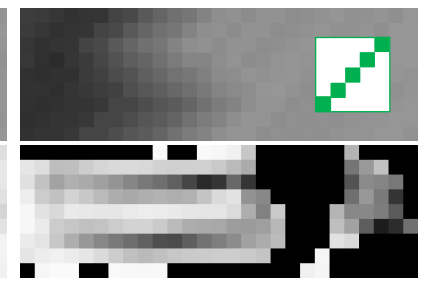

(d) Denoising along another slope

Fig. 2 EPIs (top) and confidence maps (bottom). The insets show filter kernels applied to noisy EPI.

plane image (EPI). For example, $l^{*} y^{*}(x, s)=l\left(s, t^{*}, x, y^{*}\right)$ is an EPI on the $(x, s)$ plane where $t$ and $y$ are fixed to $t^{*}$ and $y^{*}$, respectively, as shown in Fig. 1 (right). The EPI consists of many lines, each of which is a trace of an object point, and its direction (slope) corresponds to the depth of the object point. Therefore, analyzing the line direction is equivalent to estimating the depth [15]-[17], [19]-[21].

Based on this idea, Wanner and Goldluecke [15], [19] have developed a depth estimation method using structure tensor analysis. A structure tensor on an EPI $l(x, s)$ is defined as:

$$
J(x, s)=\left[\begin{array}{ll}
G *\left(l_{x} l_{x}\right) & G *\left(l_{x} l_{s}\right) \\
G *\left(l_{x} l_{s}\right) & G *\left(l_{s} l_{s}\right)
\end{array}\right],
$$

where $G *$ denotes convolution with a Gaussian filter kernel. In this paper, the filter size is fixed to the default value of [28]: a $3 \times 3$ kernel with $\sigma=1$. Symbols $l_{x}$ and $l_{s}$ denote gradients of the EPI along $x$ and $s$ directions. The dominant gradient direction $\theta(x, s)$ and its confidence $c(x, s)$ (coherence in [15], [19]) can be obtained from principle component analysis of matrix $J$.

$$
\begin{aligned}
& \theta(x, s)=\frac{1}{2} \arctan \left(\frac{2 J_{12}(x, s)}{J_{11}(x, s)-J_{22}(x, s)}\right) \\
& c(x, s)=\frac{\sqrt{\left(J_{11}(x, s)-J_{22}(x, s)\right)^{2}+4 J_{12}^{2}(x, s)}}{J_{11}(x, s)+J_{22}(x, s)},
\end{aligned}
$$

where larger $c(x, s)$ means stronger confidence. The disparity $d(x, s)$ is given by $d(x, s)=\tan \theta(x, s)$. For simplicity, we describe these processes as a function EPIAnaly $(\cdot)$. For fixed $t^{*}$ and $y^{*}$, this function is written as:

$$
\left(d^{t^{y^{*}}}(x, s), c^{t^{*} y^{*}}(x, s)\right)=\text { EPIAnaly }\left(l^{*} y^{*}(x, s)\right) \text {. }
$$

To obtain a disparity map for a specific viewpoint $\left(s^{*}, t^{*}\right)$, we perform the EPI analysis for both on $(x, s)$ and $(y, t)$ EPIs and combine the results in accordance with the point-wise confidence values.

$$
d^{s^{*} t^{*}}(x, y)= \begin{cases}d^{t^{*} y}\left(x, s^{*}\right) & c^{t^{*} y}\left(x, s^{*}\right) \geq c^{s^{*} x}\left(y, t^{*}\right) \\ d^{s^{*} x}\left(y, t^{*}\right) & \text { otherwise. }\end{cases}
$$

The disparity map $d^{s^{*} t^{*}}(x, y)$ is further refined using a fast denoising or a more sophisticated global optimization method. The former, which was used in this paper, is given as:

$$
\hat{d}^{s^{*} t^{*}}(x, y)=\arg \min _{d(x, y)} E\left(d(x, y), d^{s^{*} t^{*}}(x, y)\right)
$$

$$
\begin{aligned}
& E\left(\alpha, \alpha_{0}\right)=\int_{\Omega \subset \mathcal{R}^{2}} h(x, y)\left|D_{x y} \alpha\right|+\frac{1}{2 \lambda}\left|\alpha-\alpha_{0}\right| d \Omega \\
& h(x, y)=1-\max \left(c^{t^{*} y}\left(x, s^{*}\right), c^{s^{*} x}\left(y, t^{*}\right)\right),
\end{aligned}
$$

where $\Omega$ is the 2-D pixel domain, $\lambda$ is a smoothing strength, and $D_{x y}$ is a 2-D derivative operator. The first term of Eq. (7) penalizes non-smoothness on the disparity map, and its strength is controlled by the per pixel confidence of the initial disparity value; less confident disparities are more strongly smoothed.

\section{Proposed Method}

\subsection{Denoising an EPI with 1-D Slanted Filters}

Figure 2 illustrates an essential problem in depth estimation on an EPI and our idea as a remedy for this. As shown in (a), the noiseless EPI (top) exhibits clear line patterns, with which stable disparity estimation is possible, as indicated by the high confidence values (bottom). However, if the EPI is contaminated by noise as shown in (b), the line patterns become obscure, so we can no longer estimate disparities confidently. To solve this problem, we should somehow remove or soften the effect of noise before applying the EPI analysis.

In doing so, we focus on an inherent structure of the noiseless EPIs: the pixel values are almost the same along the intrinsic line patterns. To fully exploit this structure for denoising, we propose using a simple averaging filter whose kernel has only 1-D support, as shown in the insets of (c) and (d). Specifically, the denoising process on $l(x, s)$ is described as

$$
\operatorname{Denoise}(l(x, s) ; a)=\frac{1}{2 r+1} \sum_{\left|s^{\prime}\right| \leq r} l\left(x+a s^{\prime}, s+s^{\prime}\right),
$$

where $a$ and $r$ determine the direction (slope) and length of the filter kernel. For a non-integer pixel position, we apply linear interpolation. Different from the edge-preserving or edge-aware filters [29]-[32], our filter does not at all destroy the underlying line patterns if the lines in a local region have a constant slope and $a$ is chosen correctly. Moreover, our filter does not require fine parameter tuning in accordance with the noise level. In fact, parameter $r$ was fixed to 2 for all noise levels in our research.

In Fig. 2 (c) (d), we used two different values for $a$ in 
applying this denoising filter to the noisy EPI. When $a$ corresponds to the intrinsic line patterns in the target EPI $((c))$, we can successfully remove the noise while preserving the underlying line patterns. Meanwhile, when $a$ takes an inappropriate value $((\mathrm{d}))$, the noise can be removed, but at the same time the intrinsic line patterns are blurred out. Importantly, we can identify which value of $a$ is suitable to this EPI by observing the confidence values; they are high in (c) but low in (d). This observation leads to a simple but effective algorithm that will be presented in the next subsection.

\subsection{Algorithm}

We first determine the candidate slope values $a_{n}$ for denoising as

$$
a_{n}=a_{\min }+\frac{a_{\max }-a_{\min }}{N-1} n \quad(n=0,1, \cdots, N-1) .
$$

where $a_{n}$ changes from $a_{\min }$ to $a_{\max }$ in $N$ steps. For all pixels on the EPI $l^{*} y^{*}(x, s)$, we perform denoising along the slope $a_{n}$ as

$$
l_{n}^{t^{*} y^{*}}(x, s)=\operatorname{Denoise}\left(l^{t^{*} y^{*}}(x, s) ; a_{n}\right),
$$

followed by the structure tensor analysis as

$$
\left(d_{n}^{t^{*} y^{*}}(x, s), c_{n}^{t^{*} y^{*}}(x, s)\right)=\operatorname{EPIAnaly}\left(l_{n}^{t^{*} y^{*}}(x, s)\right) .
$$

We repeat the processes of Eqs. (11) and (12) for all the slope values $a_{n}$ and merge the results in accordance with the confidence values.

$$
\left(d^{t^{*} y^{*}}(x, s), c^{t^{*} y^{*}}(x, s)\right)=\operatorname{Merge}\left(\left\{d_{n}^{t^{*} y^{*}}(x, s), c_{n}^{t^{*} y^{*}}(x, s)\right\}\right) .
$$

The function Merge is given as follows:

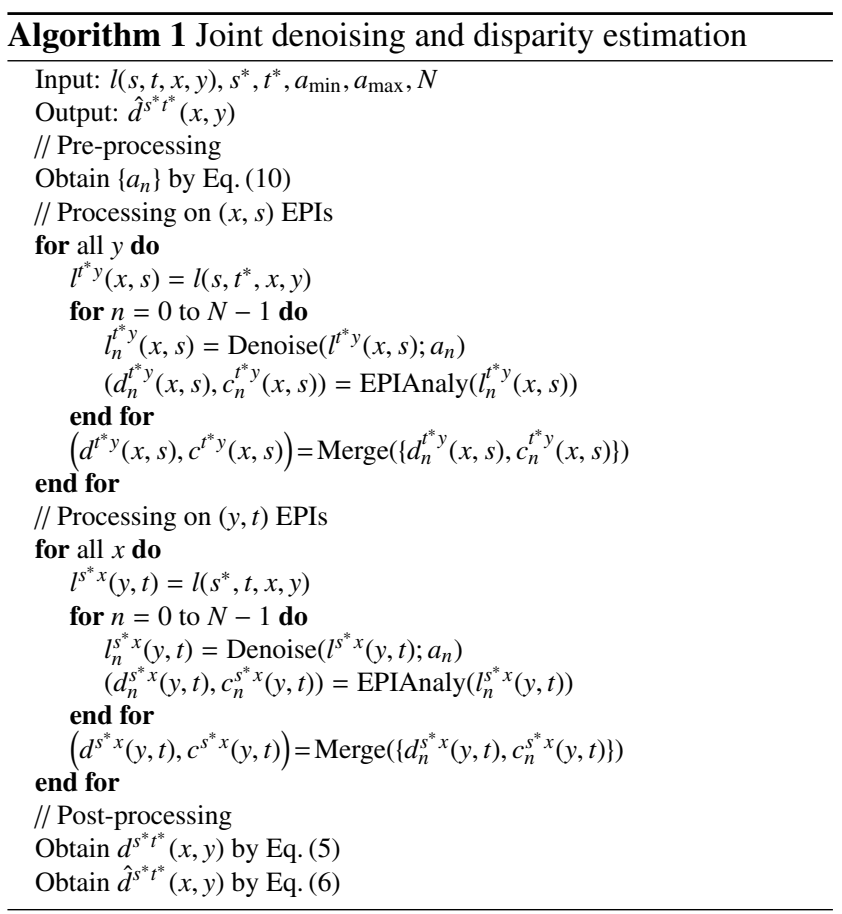

$$
\begin{aligned}
& c(x, s)=\max _{n} c_{n}(x, s) \\
& n(x, s)=\arg \max _{n} c_{n}(x, s) \\
& d(x, s)=d_{n(x, s)}(x, s) .
\end{aligned}
$$

The idea behind this merging process is that the disparity value estimated with the highest confidence over all $a_{n}$ should be preserved.

To obtain a disparity map for a given viewpoint $\left(s^{*}, t^{*}\right)$, we perform the processes of Eqs. (11)-(13) both on $(x, s)$ and $(y, t)$ EPIs. Finally, we integrate the disparity values using Eq. (5) and Eq. (6). The entire procedure of our method is described in Alg. 1.

\section{Experiments}

In Sect. 4.1, we present the effect of the parameter $N$ (in Eq. (10)). In Sects. 4.2 and 4.3, we compare our method with other denoisers and other state-of-the-art disparity estimation methods that are robust to noise. Finally, in Sect. 4.4, we apply our method to real-world inputs.

We used a PC running Ubuntu 14.04 LTS, equipped with an Intel Xeon (R) $3.10 \mathrm{GHz}$ central processing unit (CPU), 16 GB main memory, and a GeForce GTX $660 \mathrm{Ti}$ graphics processing unit (GPU). To implement our method fully utilizing the GPU, we used a continuous global optimization library (cocolib) and light field suite software, both of which were available online [28].

We used computer-generated light field datasets that are available from [33], [34]. Each light field consists of $9 \times 9$ views. Table 1 shows the disparity range of datasets. We evaluated the accuracy of the estimated disparity maps against the provided ground truth using the mean squared errors (MSE). To simulate a noisy condition, artificial zeromean Gaussian noise (the standard deviation is denoted by $\sigma)$ was added to the input light fields.

\subsection{Trade-Off between Accuracy and Processing Time}

We first evaluated the effect of the parameter $N$ in Eq. (10). We fixed $a_{\min }=-2.0$ and $a_{\max }=2.0$ because the range of slopes should cover the disparity range. We used the "buddha" dataset with the noise of $\sigma=10$. As can be seen from Fig. 3, the accuracy of the estimated disparity increases as the number of steps $N$ becomes large. However, the processing time also increases along with $N$. Considering the trade-off, we determined to use $N=5$ in the experiments later. This value will be appropriate for other datasets if

Table 1 Light field datasets used in Sect. 4

\begin{tabular}{c|c} 
Datasets & Disparity range \\
\hline \hline buddha & {$[-0.85,1.62]$} \\
\hline monasRoom & {$[-0.79,0.72]$} \\
\hline stillLife & {$[-2.71,2.62]$} \\
\hline boxes & {$[-2.20,1.40]$} \\
\hline museum & {$[-1.50,1.30]$} \\
\hline dino & {$[-1.90,1.90]$} \\
\hline
\end{tabular}


they have similar ranges of disparities, as was the case in our experiments.

\subsection{Comparison with Other Denoisers}

We next evaluated our idea of denoising along the slopes. We compared five scenarios including those using the standard denoisers before applying the EPI analysis: (i) EPI analysis on the noise-free input (noise-free), (ii) EPI analysis directly applied on the noisy input (noisy), (iii) image domain denoising followed by the EPI analysis (spatial denoising), (iv) EPI domain denoising followed by the EPI analysis (EPI denoising), and (v) our method. For all the cases, the EPI analysis was implemented with GPU acceleration.

For scenarios (iii) and (iv), we used a non-local means

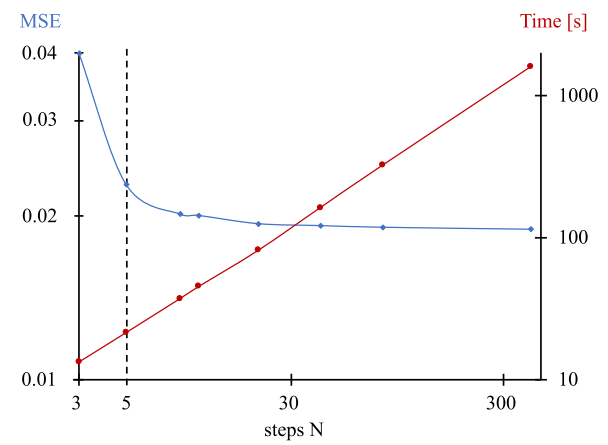

Fig. 3 Accuracy and processing time with different $N$. filter [30] implemented without GPU acceleration. We chose this filter because it has a similar order of complexity to our method, and it is also known to be edge preserving, reducing the risk of erasing the underlying line patterns while removing the noise. The kernel size and search range for the non-local means filter were set to $3 \times 3$ and $5 \times 5$ pixels. In this case, the number of neighboring pixels involved in the filtering operation for a pixel was 25 . This number was close to that of our method, where 21 neighboring pixels were used for denoising a pixel in the case of $N=5$ and $r=2$. It should be noted that in this evaluation, we only verified the idea of denoising along the slopes on an EPI in comparison with standard denoising approaches on the spatial and EPI domains under the similar order of complexity. We did not intend that our method would achieve better denoising performance than more sophisticated (but inevitably computationally-complex) methods [35], [36], because our method was designed rather simple for fast computation.

Figure 4 shows the accuracy of the disparity maps obtained at different noise levels from six datasets. Figure 5 shows disparity maps estimated with $\sigma=10.0$. It can be seen that our method produced more accurate disparity maps than the simple edge-preserving denoisers that were applied on both the image and the EPI domains.

The computational time for each scenario is presented in Fig. 6. Most of the computational time of our method was devoted to the EPI analysis operation, which was repeated $N$ times. Although this repetition induces additional computational cost, our method can still be executed in a moderate time compared to the bare EPI analysis (the noisy case).

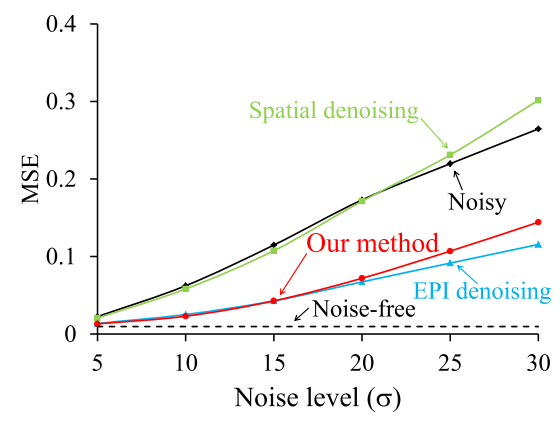

(a) buddha

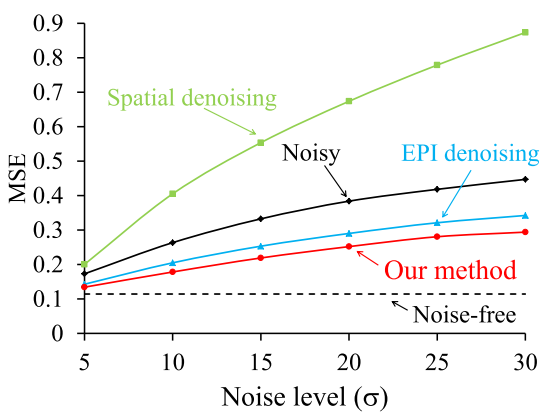

(d) boxes

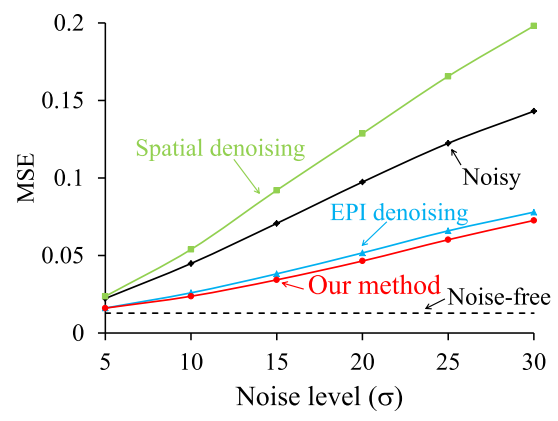

(b) monasRoom

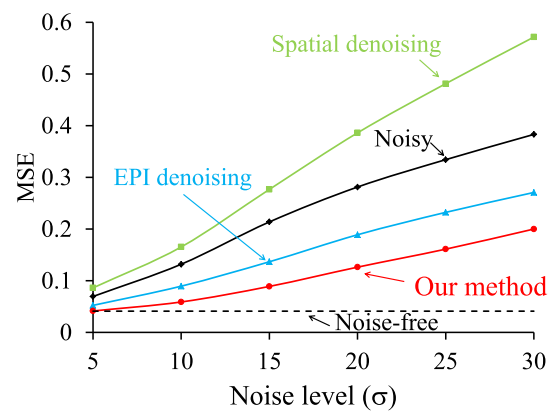

(e) museum

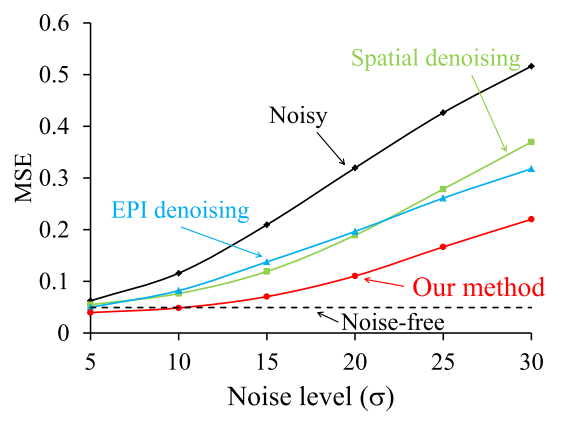

(c) stillLife

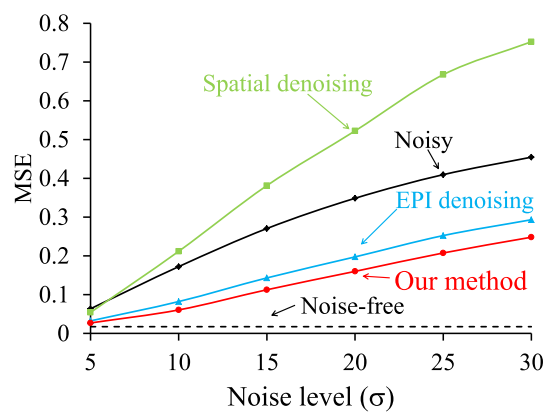

(f) dino

Fig. 4 Noise level and disparity accuracy for six datasets. 

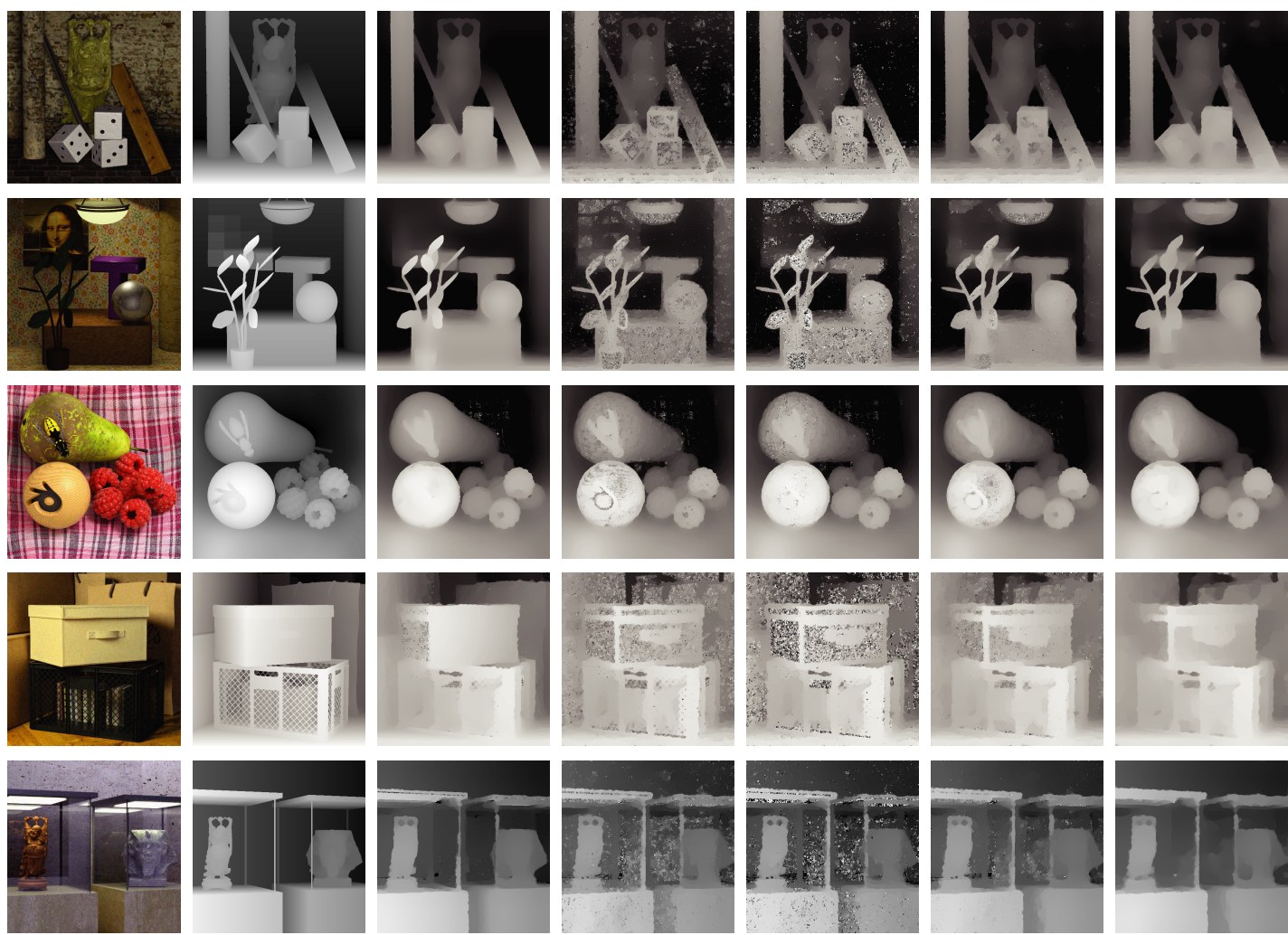

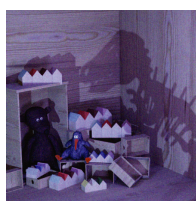

(a) Center view

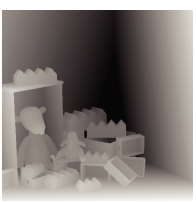

(b) Ground truth

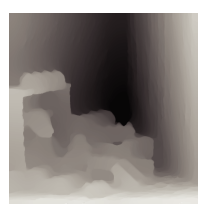

(c) Noise-free

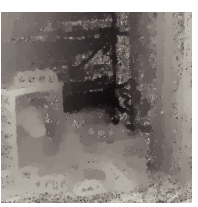

(d) Noisy

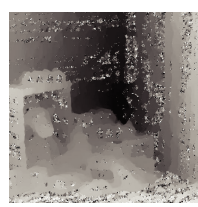

(e) Spatial denoising

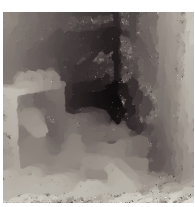

(f) EPI denoising

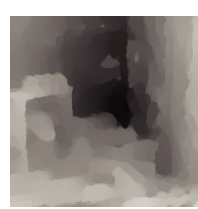

(g) Our method

Fig. 5 Visual comparison of disparity maps estimated with $\sigma=10$. From top to bottom, we used buddha, monasRoom, stillLife, boxes, museum, and dino datasets.

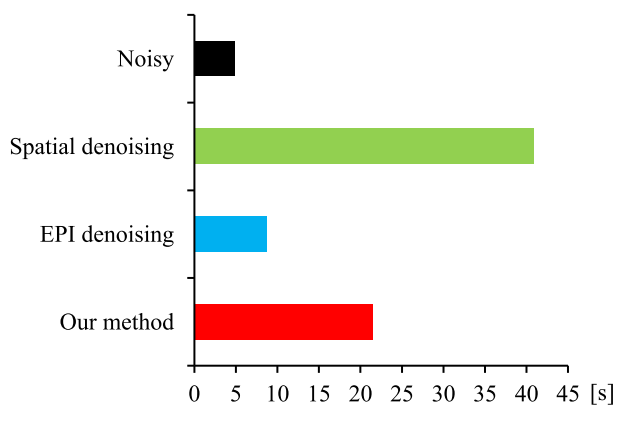

Fig. 6 Processing time for scenarios (ii)-(v).

Note also that the non-local means filter for the spatial/EPI denoising was implemented without GPU acceleration, and thus, the computational time reported here should be considered only for reference.

Another advantage of our method is that it does not require elaborate parameter configurations. In fact, we used the same parameters for our method for all the conditions in Fig. 4. Meanwhile, for the non-local means filter, we adjusted the parameter ( $h$ in [30]) for the target noise level

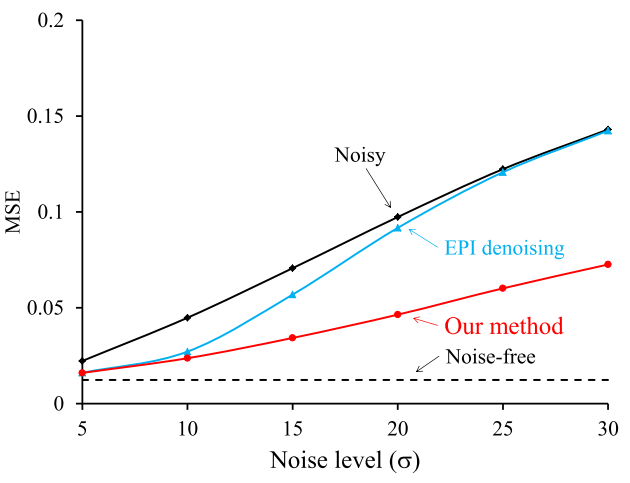

Fig. 7 Noise level and disparity accuracy with the "monasRoom" dataset. This graph is different from Fig. 4 (b) in that the noise level was simply assumed to be $\sigma=5$ for EPI denoising.

to achieve a good performance. For instance, as shown in Fig. 4 (b), EPI denoising performed well if it was provided with the target noise levels. However, as shown in Fig. 7, its performance became poor if the noise level was simply assumed to be $\sigma=5.0$. Note that in practical applications, we rarely know the exact noise level. 


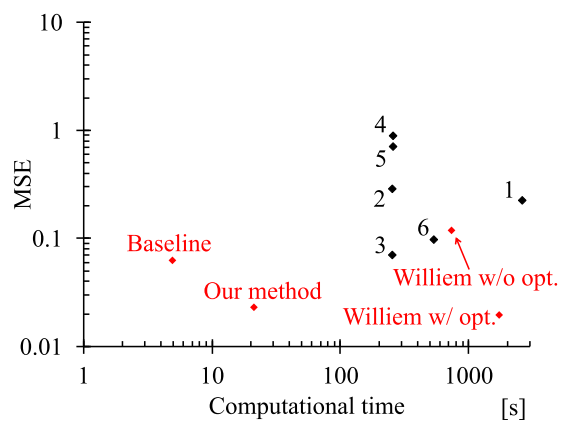

(a) buddha $(\sigma=10)$

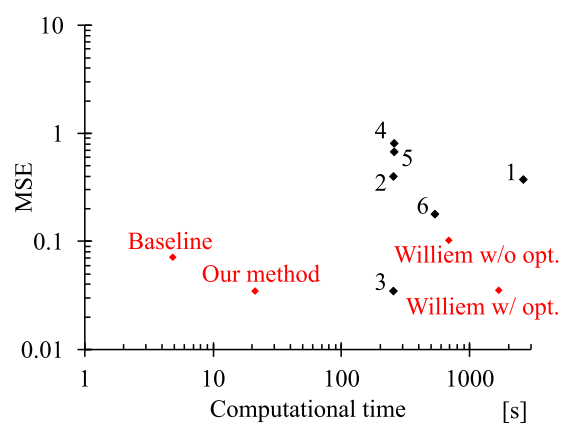

(b) monasRoom $(\sigma=15)$

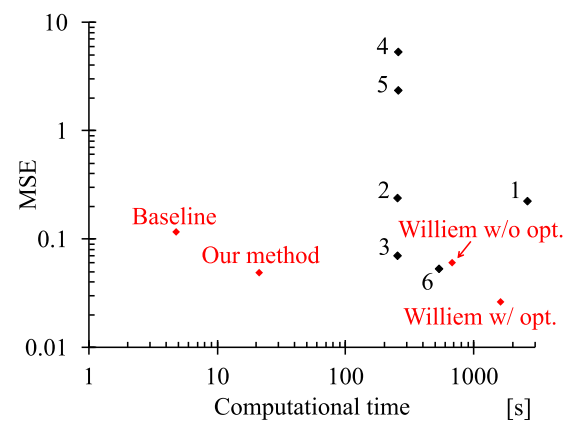

(c) stillLife $(\sigma=10)$

Fig. 8 Computational time and disparity accuracy. See Table 2 for legends.
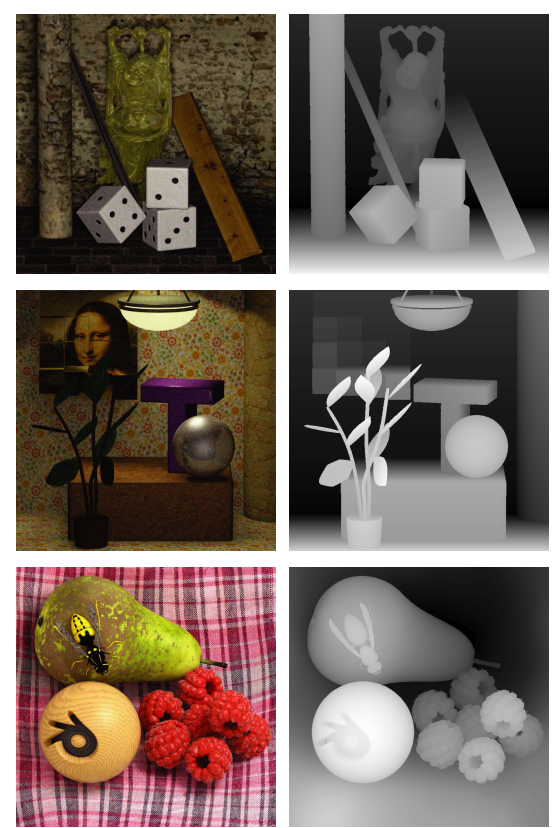

(a) Center view

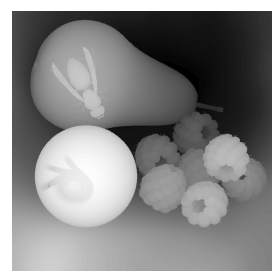

(b) Ground truth
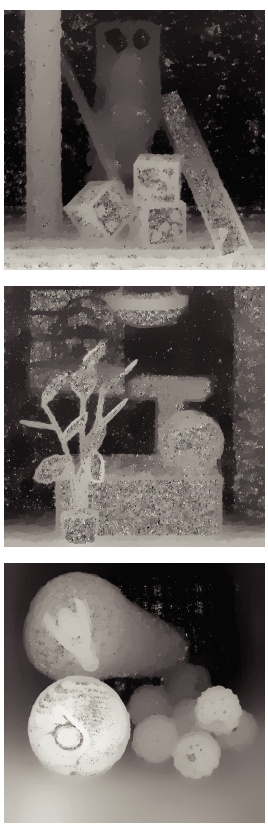

(c) EPI analysis [19]
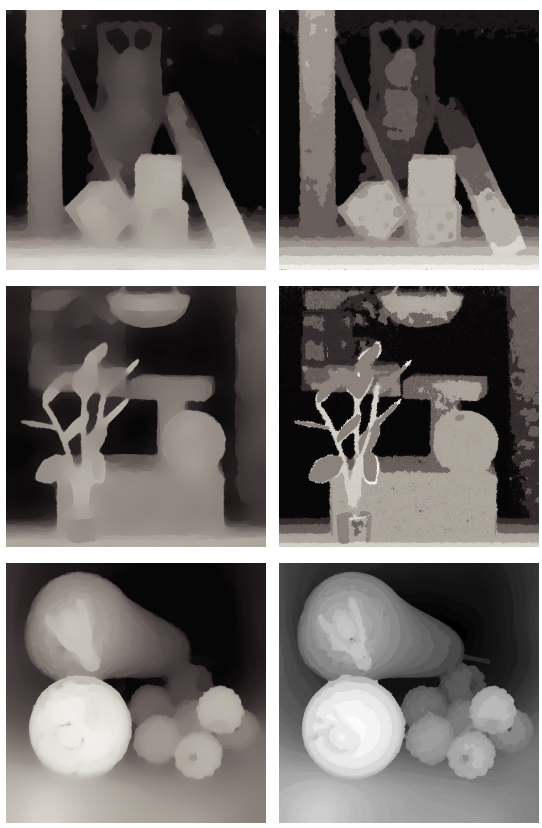

(d) Our method

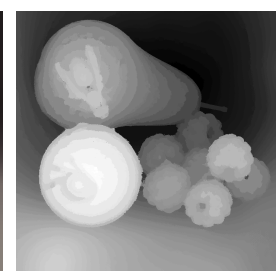

(e) Williem w/ opt. [25]

Fig. 9 Visual comparison of disparity maps. From top to bottom, the noise level was $\sigma=10, \sigma=15$, and $\sigma=10$.

Table 2 Legends for Fig. 8

\subsection{Comparison with Other Disparity Estimation Methods}

We compared the performance of our method with other state-of-the-art methods in terms of accuracy and computational time, as shown in Fig. 8. We used three datasets with non-identical noise levels, to show that our method did not overfit a specific dataset or a noise level. In addition to the baseline EPI analysis [19], dubbed as "baseline", we obtained an authors' implementation of Williem's method [25], [26], which was stated as being robust to noise. Their method was tested with and without global optimization, which is time-consuming but can improve the accuracy. We plotted all the values obtained with our environment in red. Moreover, we added the plots in black for other stateof-the-art methods on the basis of the reported values in [25] (see Table 2 for the legends) ${ }^{\dagger}$. The method indicated by

${ }^{\dagger}$ For the methods of [13], [14], only the total computational time was reported while the accuracy was evaluated individually for the defocus and correspondence costs.

\begin{tabular}{l|c} 
Methods & Legends \\
\hline \hline Jeon's data cost [11] & 1 \\
\hline Tao's correspondence cost [13] & 2 \\
\hline Tao's defocus cost [13] & 3 \\
\hline Wang's correspondence cost [14] & 4 \\
\hline Wang's defocus cost [14] & 5 \\
\hline Williem w/o optimization [25] & 6 \\
\hline
\end{tabular}

"6" is the same as "Williem w/o opt.", but the MSE values were slightly different, seemingly, due to some difference of the experimental conditions. It was reported in [25] that they used a PC with an Intel i7 $47703.4 \mathrm{GHz}$ CPU and 12 GB main memory, which resulted in faster computation than ours for the same method.

As can be seen from these graphs, our method achieved good accuracy compared to the state-of-the-art methods. Although our method induced an additional computational cost in the baseline EPI analysis [19], it was still a few orders of magnitude faster than the other methods. It should 

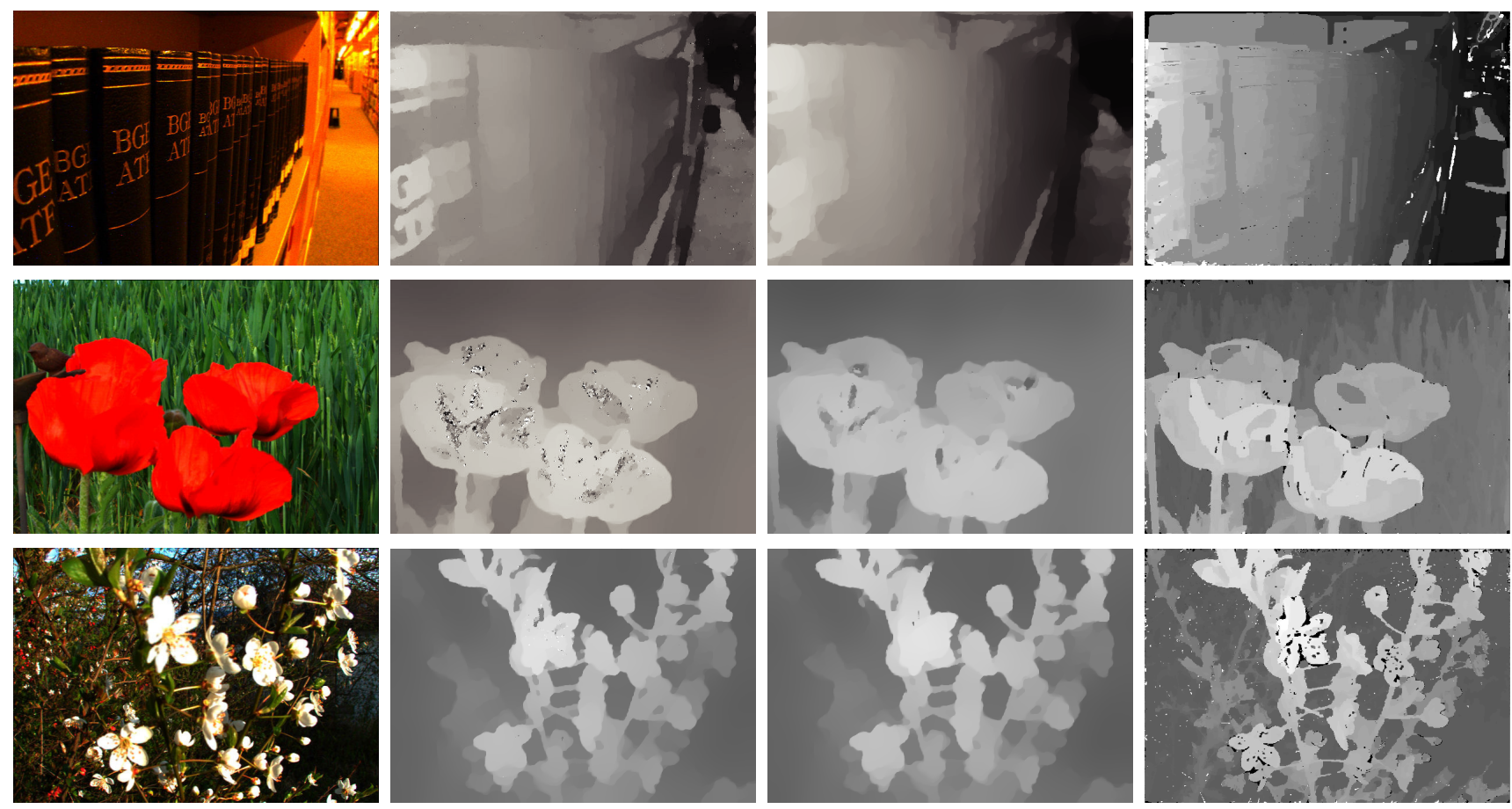

(a) Center view

(b) EPI analysis [19]

(c) Proposed

(d) Williem w/ opt. [25]

Fig. 10 Real world results with "Books", "Poppies" and "Mirabelle" datasets [37] from top to bottom.

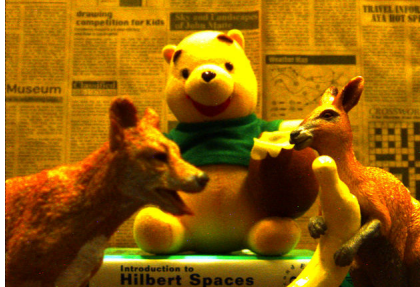

(a) Center view

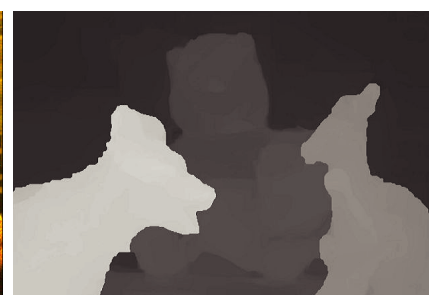

(b) Lytro Desktop

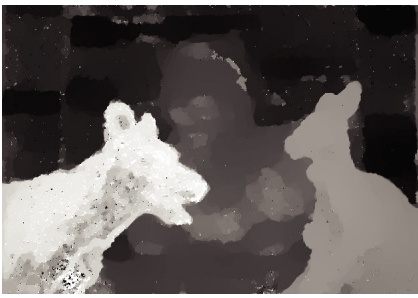

(c) EPI analysis [19]

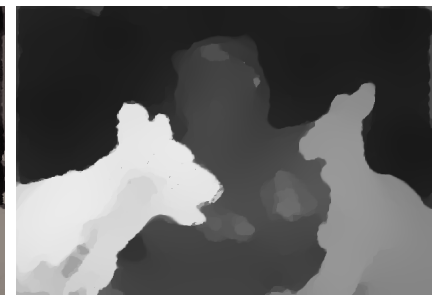

(d) Our method

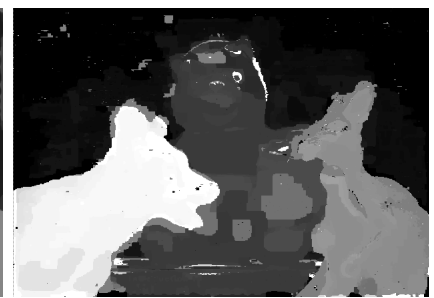

(e) Williem w/ opt. [25]

Fig. 11 Real world results with a light field obtained by ourselves.

be noted that most of the methods did not put importance on the computational time; the baseline EPI analysis and our method were implemented with GPU acceleration, but it seems that the other methods were not optimized for speed, nor implemented with GPU acceleration.

Figure 9 shows disparity maps including ones estimated by Williem's method. Our disparity maps are visually better than those of Williem's method.

\subsection{Real-world Results}

We also tested several real-world light field datasets, which are inherently somewhat noisy depending on the shooting condition, to validate the effectiveness of our method. We used some datasets taken by Lytro Illum cameras that were available from [37]. We estimated disparity maps with the baseline EPI analysis [19], our proposed method, and 
Williem's method [25], [26], and presented the results in Fig. 10. We also used a light field taken by ourselves using a Lytro Illum camera, whose disparity ranges between the neighboring viewpoints were from -1.5 to 1.5 . The results are presented in Fig. 11, where a depth map obtained with Lytro Desktop ver. 5.0.1 is also presented for reference. As shown in those results, our method produced visually compelling disparity maps even from noisy real-world light fields.

\section{Conclusion}

We addressed the problem of fast and accurate disparity estimation from a dense but noisy light field. In constructing our method, we incorporated a fast denoising scheme using simple 1-D slanted filters into the fast disparity estimation framework in the EPI domain. Experimental results show that our method can achieve good accuracy with much less computational time compared to some state-of-the-art methods. In future work, we will extend our method to larger disparity ranges as in [20] and less-textured objects that are common in natural scenes.

\section{References}

[1] C. Zhang and T. Chen, "A self-reconfigurable camera array," Proc. Eurographics Conference on Rendering Techniques (EGSR), pp.243-254, 2004.

[2] R. Ng, M. Levoy, M. Brédif, G. Duval, M. Horowitz, and P. Hanrahan, "Light field photography with a hand-held plenoptic camera," Stanford Tech Report, vol.2, pp.1-11, 2005.

[3] B. Wilburn, N. Joshi, V. Vaish, E.V. Talvala, E. Antunez, A. Barth, A. Adams, M. Horowitz, and M. Levoy, "High performance imaging using large camera arrays," ACM Trans. Graphics, vol.24, no.3, pp.765-776, July 2005.

[4] Y. Taguchi, K. Takahashi, and T. Naemura, "Real-time all-in-focus video-based rendering using a network camera array," Proc. 3DTV Conference: The True Vision-Capture, Transmission and Display of 3D Video, pp.241-244, 2008.

[5] K. Marwah, G. Wetzstein, Y. Bando, and R. Raskar, "Compressive light field photography using overcomplete dictionaries and optimized projections," ACM Trans. Graphics, vol.32, no.4, July 2013.

[6] M. Okutomi and T. Kanade, "A multiple-baseline stereo," IEEE Trans. Pattern Anal. Mach. Intell., vol.15, no.4, pp.353-363, April 1993.

[7] S.B. Kang, R. Szeliski, and J. Chai, "Handling occlusions in dense multi-view stereo," Proc. IEEE Computer Society Conference on Comput. Vis. Pattern Recognit. (CVPR), pp.103-110, 2001.

[8] C. Strecha, R. Fransens, and L.V. Gool, "Combined depth and outlier estimation in multi-view stereo," Proc. IEEE Computer Society Conference on Comput. Vis. Pattern Recognit. (CVPR), pp.23942401, 2006.

[9] S.M. Seitz, B. Curless, J. Diebel, D. Scharstein, and R. Szeliski, "A comparison and evaluation of multi-view stereo reconstruction algorithms," Proc. IEEE Computer Society Conference on Comput. Vis. Pattern Recognit. (CVPR), pp.519-528, 2006.

[10] N.D.F. Campbell, G. Vogiatzis, C. Hernández, and R. Cipolla, "Using multiple hypotheses to improve depth-maps for multi-view stereo," Proc. European Conference on Computer Vision (ECCV), LNCS, vol.5302, pp.766-779, 2008.

[11] H.G. Jeon, J. Park, G. Choe, J. Park, Y. Bok, Y.W. Tai, and I.S. Kweon, "Accurate depth map estimation from a lenslet light field camera," Proc. IEEE Computer Society Conference on Comput. Vis.
Pattern Recognit. (CVPR), pp.1547-1555, 2015.

[12] J. Navarro and A. Buades, "Robust and dense depth estimation for light field images," IEEE Trans. Image Process., vol.26, no.4, pp.1873-1886, April 2017.

[13] M.W. Tao, P.P. Srinivasan, J. Malik, S. Rusinkiewicz, and R. Ramamoorthi, "Depth from shading, defocus, and correspondence using light-field angular coherence," Proc. IEEE Conference on Comput. Vis. Pattern Recognit. (CVPR), pp.1940-1948, 2015.

[14] T.C. Wang, A.A. Efros, and R. ramamoorthi, "Occlusion-aware depth estimation using light-field cameras," Proc. IEEE Int. Conf. Computer Vision (ICCV), pp.3487-3495, 2015.

[15] S. Wanner and B. Goldluecke, "Globally consistent depth labeling of 4D light fields," Proc. IEEE Conference on Comput. Vis. Pattern Recognit., pp.41-48, 2012.

[16] M. Diebold and B. Goldlucke, "Epipolar plane image refocusing for improved depth estimation and occlusion handling," Proc. Annual Workshop on Vision, Modeling and Visualization, pp.145-152, 2013.

[17] J. Li, M. Lu, and Z.N. Li, "Continuous depth map reconstruction from light fields," IEEE Trans. Image Process., vol.24, no.11, pp.3257-3265, Nov. 2015.

[18] I. Tosic and K. Berkner, "Light field scale-depth space transform for dense depth estimation," Proc. IEEE Conference on Comput. Vis. Pattern Recognit. Workshops, pp.435-442, 2014.

[19] S. Wanner and B. Goldluecke, "Variational light field analysis for disparity estimation and super-resolution," IEEE Trans. Pattern Anal. Mach. Intell. , vol.36, no.3, pp.606-619, March 2014.

[20] T. Suzuki, K. Takahashi, and T. Fujii, "Sheared EPI analysis for disparity estimation from light fields," IEICE Trans. Inf. \& Syst., vol.E100-D, no.9, pp.1984-1993, Sept. 2017.

[21] R.C. Bolles, H.H. Baker, and D.H. Marimont, "Epipolar-plane image analysis: An approach to determining structure from motion," Int. J. Comput. Vis., vol.1, no.1, pp.7-55, March 1987.

[22] A. Criminisi, S.B. Kang, R. Swaminathan, R. Szeliski, and P. Anandan, "Extracting layers and analyzing their specular properties using epipolar-plane-image analysis," Computer Vision and Image Understanding, vol.97, no.1, pp.51-85, Jan. 2005.

[23] J. Berent and P.L. Dragotti, "Segmentation of epipolar-plane image volumes with occlusion and disocclusion competition," Proc. IEEE Workshop on Multimedia Signal Processing, pp.182-185, 2006.

[24] R. GmbH, "Lytro - Home." http://lytro.com/, 2018.

[25] W. Williem and I.K. Park, "Robust light field depth estimation for noisy scene with occlusion," Proc. IEEE Conference on Comput. Vis. Pattern Recognit. (CVPR), 2016.

[26] W. Williem, I.K. Park, and K.M. Lee, "Robust light field depth estimation using occlusion-noise aware data costs," IEEE Trans. Pattern Anal. Mach. Intell., vol.40, no.10, pp.2484-2497, Oct. 2018.

[27] G. Houben, S. Fujita, K. Takahashi, and T. Fujii, "Fast and robust disparity estimation for noisy light fields," Proc. IEEE Int. Conf. Image Process., pp.1-5, 2018.

[28] B. Goldluecke, "Cocolib / light field suite." http://sourceforge.net/p/ cocolib/home/Home/, 2018.

[29] C. Tomasi and R. Manduchi, "Bilateral filtering for gray and color images," Proc. Int. Conf. Computer Vision (ICCV), pp.839-846, Jan. 1998 .

[30] A. Buades, B. Coll, and J.M. Morel, "A non-local algorithm for image denoising," Proc. IEEE Computer Society Conference on Comput. Vis. Pattern Recognit. (CVPR), pp.60-65, June 2005.

[31] E.S.L. Gastal and M.M. Oliveira, "Domain transform for edgeaware image and video processing," ACM Trans. Graphics, vol.30, no.4, Article No. 69, July 2011.

[32] K. He, J. Sun, and X. Tang, "Guided image filtering," IEEE Trans. Pattern Anal. Mach. Intell., vol.35, no.6, pp.1397-1409, June 2013.

[33] H.C.I., "Light field analysis." http://klimt.iwr.uni-heidelberg.de/ HCI/Research/\LightField/vmv-benchmark-2013.php.

[34] H.C.I., " $4 \mathrm{~d}$ light field dataset." http://hci-lightfield.iwr.uni-heidelberg. $\mathrm{de} /$. 
[35] M. Maggioni, G. Boracchi, A. Foi, and K. Egiazarian, "Video denoising, deblocking, and enhancement through separable 4-D nonlocal spatiotemporal transforms," IEEE Trans. Image Process., vol.21, no.9, pp.3952-3966, Sept. 2012.

[36] A. Sepas-Moghaddam, P.L. Correia, and F. Pereira, "Light field denoising: exploiting the redundancy of an epipolar sequence representation," 2016 3DTV-Conference: The True Vision - Capture, Transmission and Display of 3D Video (3DTV-CON), pp.1-4, 2016.

[37] M.S.P. GROUP, "JPEG pleno database: EPFL light-field data set." https://jpeg.org/plenodb/lf/epfl/, 2018.

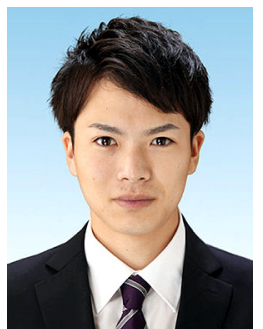

Gou Houben received a B.E. degree in electrical and electronic engineering and information engineering from Nagoya University, Nagoya, Japan, in 2017 and an M.E. degree in information and communication engineering from Nagoya University, Nagoya, Japan, in 2019. When he was a student, his research topic was disparity estimation from a light field.

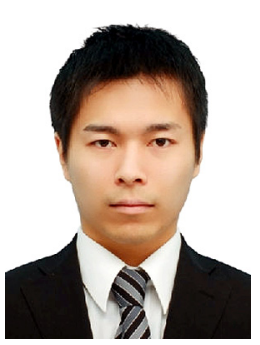

Shu Fujita received B.E. and M.E. degrees in information engineering from Nagoya Institute of Technology, Nagoya, Japan, in 2014 and 2016 and a Ph.D. degree in electrical engineering and computer science from Nagoya University, Nagoya, Japan, in 2019. When he was a student, his research topic was light field acquisition and processing.

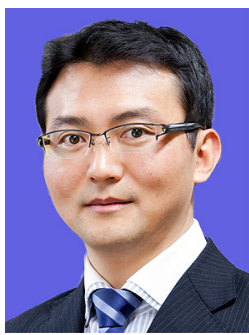

Keita Takahashi received his B.E., M.S., and $\mathrm{Ph} . \mathrm{D}$. degrees in information and communication engineering from the University of Tokyo, Japan, in 2001, 2003, and 2006. He was a project assistant professor at the University of Tokyo from 2006-2011 and was an assistant professor at the University of ElectroCommunications, Japan from 2011-2013. He is currently an associate professor at the Graduate School of Engineering, Nagoya University, Japan. His research interests include light field processing, image-based rendering, computational photography, and 3D display. He is a member of IEEE SPS \& CS, ITE, IPSJ, and IEICE.

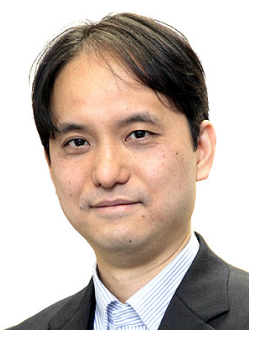

Toshiaki Fujii received B.E., M.E., and Dr.E. degrees in electrical engineering from the University of Tokyo in 1990, 1992, and 1995. From 1995, he has been with the Graduate School of Engineering, Nagoya University apart from 2008 to 2010, when he was with the Graduate School of Science and Engineering, Tokyo Institute of Technology. He is currently a professor in the Graduate School of Engineering, Nagoya University. His current research interests include multi-dimensional signal processing, multi-camera systems, multi-view video coding and transmission, freeviewpoint television, and their applications. 